\title{
Measurement of soil electrical conductivity based on direct digital synthesizer (DDS) and digital oscilloscope
}

\author{
Xiaoshuai Pei ${ }^{1}$, Chao Meng ${ }^{2}$, Minzan $\mathrm{Li}^{1 *}$, Wei Yang ${ }^{2}$, Peng Zhou ${ }^{1}$ \\ (1. Key Laboratory of Modern Precision Agriculture System Integration Research, Ministry of Education, China Agricultural University, \\ Beijing 100083, China; 2. Key Laboratory of Agricultural Information Acquisition Technology, Ministry of Agriculture and Rural Affairs, \\ China Agricultural University, Beijing 100083, China)
}

\begin{abstract}
A soil electrical conductivity (EC) measurement system based on direct digital synthesizer (DDS) and digital oscilloscope was developed. The system took the "current-voltage four-electrode method" as the design principal and adopted a six-pin structure of the probe, two center pins to measure the soil EC in shallow layer, two outside pins to measure the soil EC in deep layer, and two middle pins for inputting the driving current. A signal generating circuit using DDS technology was adopted to generate sine signals, which was connected with the two middle pins. A digital oscilloscope was used to record and store the two soil output signals with noises in microseconds, which were from the two center pins and two outside pins, respectively. Then a digital bandpass filter was used to filter the soil output signals recorded by the digital oscilloscope. Compared with the traditional analog filter circuit, the digital filter could filter out the noises of all frequency except for the frequency of the excitation source. It could improve the effect of filtering and the accuracy of the soil EC measurement system. The DDS circuit could provide more stable sine signals with larger amplitudes. The use of digital oscilloscope enables us to analyze the soil output signals in microseconds and measure the soil EC more accurately. The new soil EC measurement system based on DDS and digital oscilloscope can provide a new effective tool for soil sensing in precision agriculture.
\end{abstract}

Keywords: soil electrical conductivity, direct digital synthesizer, digital oscilloscope, precision agriculture, current-voltage four-electrode method

DOI: $10.25165 /$ j.ijabe.20191206.4840

Citation: Pei X S, Meng C, Li M Z, Yang W, Zhou P. Measurement of soil electrical conductivity based on direct digital synthesizer (DDS) and digital oscilloscope. Int J Agric \& Biol Eng, 2019; 12(6): 162-168.

\section{Introduction}

Traditional agriculture management adopts a unified management strategy for the farmland. In that farming system, massive fertilizers and pesticides are applied to ensure the yield. On the other hand, the utilization of massive fertilizers and pesticides in the farmland has led to many problems such as soil compaction, environmental pollution, and food safety problems ${ }^{[1,2]}$. Contrary to the traditional agriculture, Precision Agriculture ${ }^{[3,4]}$ differentiates agriculture management based on the soil nutrient status and crop growth conditions of different locations in the farmland and can increase yield with less input ${ }^{[5,6]}$. Rapid and accurate access to soil parameters is one of the bases of Precision Agriculture $^{[7,8]}$. Soil electrical conductivity (EC) is an important soil parameter and has a relationship with several soil properties such as soil moisture, soil salinity, soil texture, cation exchange capacity (CEC), and organic carbon content. The measurements

Received date: 2018-12-07 Accepted date: 2019-10-25

Biographies: Xiaoshuai Pei, PhD Candidate, research interests: agricultural informatization, Email: xiaoshuaipei@163.com; Chao Meng, Master Candidate, research interests: agricultural engineering, Email: mc6663@cau.edu.cn. Minzan Li, PhD, Professor, research interests: precision agriculture, Email:limz@cau.edu.cn; Wei Yang, Associate Professor, research interests: agricultural informatization, Email: cauyw@cau.edu.cn; Peng Zhou, PhD Candidate, research interests: agricultural engineering, Email: zhoupeng@ cau.edu.cn.

*Corresponding author: Minzan Li, PhD, Professor, research interest: precision agriculture. China Agricultural University, Beijing 100083, China. Tel: +86-10-62737924, Email: limz@cau.edu.cn. of the soil EC can reflect the soil status and provide a basis for implementing Precision Agriculture ${ }^{[9,10]}$.

The measurement methods of the soil EC could be broadly classified into two categories, electromagnetic induction (EMI) method and current-voltage four-electrode method. In the electromagnetic induction method, soil EC is obtained by measuring the corresponding electromagnetic field intensity of soil. It could measure soil EC of different depth and has the advantage of no contact with soil. Several soil EC detectors based on the EMI method have become commercial products, such as EM38 (Geonics Limited, Ontario, Canada), TSM (Geoprospectors GmbH, Traiskirchen, Austria). On the other hand, although those products have higher accuracy, the high price makes them unavailable to farmers. The current-voltage four-electrode method is a contact measurement method. Two electrodes are used to conduct the driving current to soil and the other two electrodes are used to detect the output signals (voltage) to calculate soil EC ${ }^{[11]}$. It has a simple structure and less affected by electromagnetic noises ${ }^{[12]}$. Research has shown that the two measurements of the soil EC have similar results ${ }^{[13]}$. The direct contact method is widely used for its robust construction, no interference from metal materials, and avoid daily calibration ${ }^{[14]}$. We have developed a vehicular soil electrical conductivity monitoring system based on the current-voltage four-electrode method, which can work together with ploughing ${ }^{[15]}$.

The soil EC measurement based on the "current-voltage four-electrode method" usually adopts an analog circuit to generate an AC current and an analog filter circuit to filter output signals. A voltage effective value conversion circuit is used to convert soil 
output signals into DC signals, which are converted into digital values by the $\mathrm{AD}$ conversion circuit ${ }^{[15-18]}$. The results of $\mathrm{AD}$ conversion are used for calculating soil EC. Although the traditional system could measure soil EC conveniently, there are still several disadvantages. For example, the limitation of the voltage effective value conversion circuit and $\mathrm{AD}$ conversion circuit's conversion frequency lead to the loss of detail information of the soil output signals, which could be used for analyzing soil EC further. It is difficult to evaluate the signal-to-noise ratio (SNR) of the output signals and the measurement accuracy of the traditional soil EC measurement system. It is also difficult to evaluate the effect of the filter circuit. If the noises are not filtered by the filter circuit, they would affect the accuracy of the traditional soil EC measurement system. Furthermore, the current value of the driving current signal generated by the analog circuit is considered as a constant value, which is easily affected by the temperature, humidity and electromagnetic noises in fact. It is difficult to evaluate the filtering effect of the analogy filter circuit and the SNR of soil output signals under farmland conditions. In response to these problems, a soil EC measurement system based on DDS and digital oscilloscope was developed, which could solve those problems effectively and improve measurement accuracy.

Direct Digital Synthesizer (DDS) is a type of frequency synthesizer used for creating arbitrary waveforms from a single, fixed-frequency reference clock. It can be used as signal generators, local oscillators in communication systems, function generators, mixers, modulators, sound synthesizers and the part of a digital phase-locked loop ${ }^{[19]}$. A basic DDS consists of a frequency reference, a numerically controlled oscillator, and a digital-to-analog converter (DAC). Digital oscilloscope could record the soil output signals at the frequency of $200 \mathrm{MHz}$, which could provide detailed information for in-depth analysis.

This study aims to develop a high accuracy measurement system of soil EC. Hence, we decided to develop a new kind of soil EC measurement system using a DDS module and a digital oscilloscope. It could solve the problems of the traditional soil EC measurement system. In the developed system, the traditional analog signal generation circuit will be replaced by a DDS circuit, which can greatly improve the accuracy and the stability of the driving current. The current value of the input signal is recorded for calculating soil EC, which can eliminate the influence of current fluctuation on soil EC. The voltage effective value conversion circuit will be replaced by the digital oscilloscope since it can collect much more information. The collected data can be used for calculating soil EC, analyzing noises and the SNR in-depth. A digital bandpass filter is used to replace the analog filter circuit so that the filtering effect can be evaluated according to the waveform of the filtered signals.

\section{Materials and methods-Development of the soil EC system based on DDS and digital oscilloscope}

\subsection{Soil EC measurement system based on DDS and digital oscilloscope}

The probe is an important part of the soil EC measurement system. Since the purpose of this study is to improve the measurement accuracy of soil EC by using DDS and digital oscilloscope, we didn't consider any improvement on the probe structure and decided to adopt a six-pin structure of the probe based on current-voltage four-electrode method ${ }^{[15]}$. As shown in Figure 1 , pin $\mathrm{J}$ and pin $\mathrm{K}$ are the electrodes for input signal, pin $\mathrm{M}$ and pin $\mathrm{N}$ are used to measure soil $\mathrm{EC}$ in shallow layer $\left(\sigma_{s}\right)$, and the pin $\mathrm{P}$ and pin Q are used to measure soil EC in deep layer $\left(\sigma_{d}\right)$.

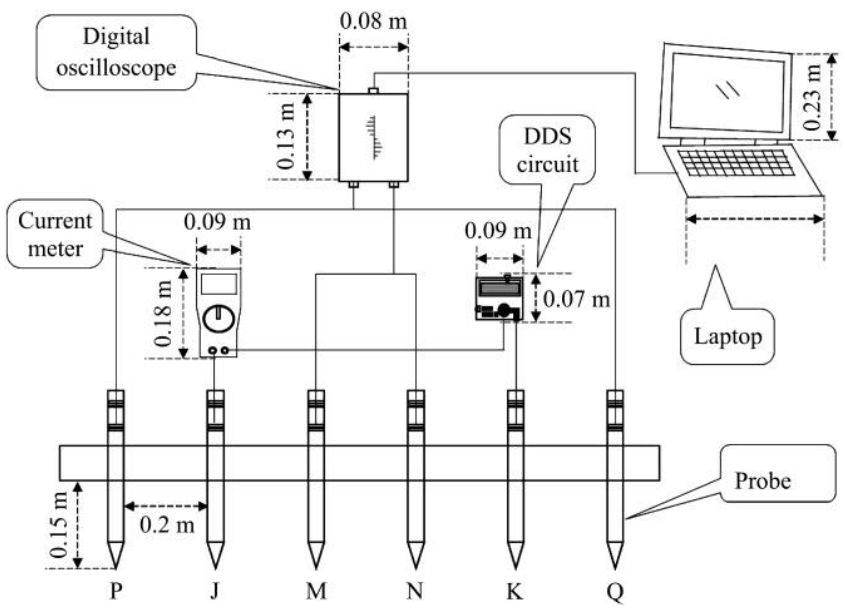

Figure 1 Illustration of the soil EC system

The distance between pin $\mathrm{P}$ and pin $\mathrm{Q}$ is $1 \mathrm{~m}$. The distance between two adjacent pins is $0.2 \mathrm{~m}$. The length of the copper pin tip is $0.15 \mathrm{~m}$. The dimension of the digital oscilloscope, DDS module and laptop were also labeled.

The calculation formula of $\sigma_{s}$ is shown as the following ${ }^{[9]}$ :

$$
\begin{aligned}
\sigma_{s} & =\frac{\left(\frac{1}{d_{J M}}-\frac{1}{d_{J N}}\right)-\left(\frac{1}{d_{K M}}-\frac{1}{d_{K N}}\right)}{2 \pi} \frac{I}{V_{M N}} \\
& \approx 0.796 \times \frac{I}{V_{M N}}
\end{aligned}
$$

And the calculation formula of $\sigma_{d}$ is shown as the following ${ }^{[9]}$.

$$
\begin{aligned}
\sigma_{d} & =\frac{\left(\frac{1}{d_{J P}}-\frac{1}{d_{J Q}}\right)-\left(\frac{1}{d_{K P}}-\frac{1}{d_{K Q}}\right)}{2 \pi} \frac{I}{V_{P Q}} \\
& \approx 1.194 \times \frac{I}{V_{P Q}}
\end{aligned}
$$

where, $\sigma$ is soil electrical conductivity, $\mathrm{S} / \mathrm{m} ; d$ is the distance between the two probes, $\mathrm{m} ; V_{M N}$ is the voltage between probe $M$ and the probe $N, \mathrm{~V} ; I$ is the current of the input signal, A; In the traditional soil EC measurement system, $I$ is considered as a constant value. However, in the developed system $I$ is considered as a variety to eliminate the influence of the input signal's fluctuation.

The overall design's comparison of the traditional soil EC measurement system and the system based on DDS and digital oscilloscope is shown in Figure 2. In the traditional system (Figure 2a), the input signal is generated by an analog circuit. It contains obvious noises and the amplitude is relatively small. The output signals are also processed by analog circuit and the frequency of the voltage effective value conversion and $\mathrm{AD}$ conversion is slow. In addition, there is no way to evaluate the SNR of the output signals and the effect of the analog filter circuit.

In response to the above questions, a new soil EC measurement system was developed (Figure 2b) which based on DDS and digital oscilloscope. The developed system consists of a DDS signal generation circuit, a multimeter, two input electrodes, four output electrodes, a digital oscilloscope, a digital filter, a PC and other components. The DDS signal generation circuit generates a sine signal with stable waveform and a larger amplitude conducts it into the soil through the input electrodes. The multimeter is used to measure the current value of the sine signal, which is used to 
eliminate the impact of the input signal fluctuations on measurement results. The soil output signals are collected and recorded by the digital oscilloscope along with the environmental noises. The maximum sampling frequency of the digital oscilloscope is $200 \mathrm{MHz}$, which can record the very detailed information. The mixed soil output signals and environment noises can be visually observed and the influence of the noises can be evaluated by analyzing the stored data. The digital bandpass filter consists of Chebyshev type I digital filters. The waveform of the output signals after filtering can be used for evaluating the effect of the digital filter. The average effective value of the output signal is used as the measurement result for calculating soil $\mathrm{EC}$, which can reduce the influence of the noises.

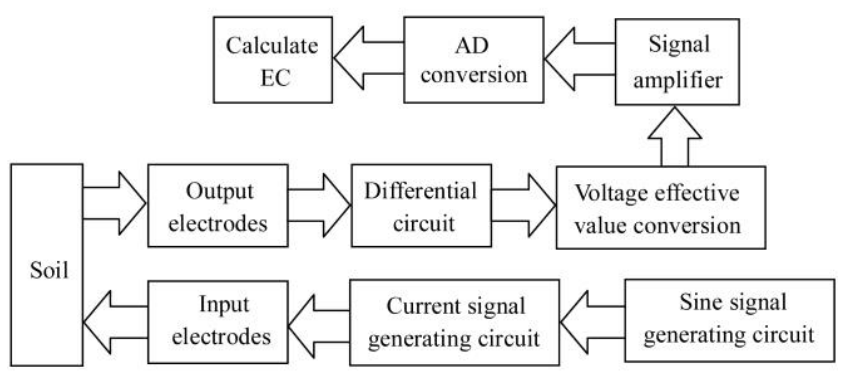

a. The overall design of the traditional soil EC measurement system

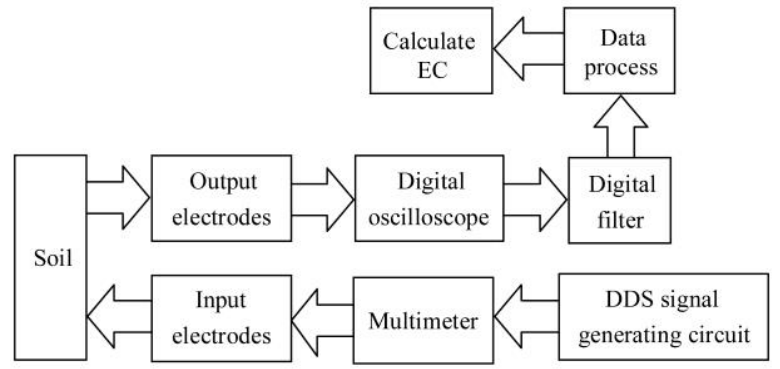

b. The overall design of the soil EC measurement system based on DDS and digital oscilloscope

Figure 2 Comparison of the overall design of the traditional soil EC system and the developed soil EC system

The developed system improves the measurement accuracy from the input signal, output signals sampling frequency, filtering effect, reducing noise by averaging and evaluating SNR of the signals. The detailed stored data also provide the feasibility of analyzing soil EC and noises further.

\subsection{DDS signal generation circuit and multimeter}

Compared with the excitation source generation circuit of the traditional soil EC measurement system, the DDS signal generation circuit has the advantages of larger amplitude, better stability and flexible operation. DDS works on the basis of the sampling theorem. Firstly, the target signal waveform is sampled and the sampled value is stored in the memory as a lookup table. In use, the value in the table is read and converted into an analog signal by a DA converter. Therefore, the saved waveform is re-synthesized.

As shown in Figure 3, the DDS signal generation circuit in the system is based on AVR microcontroller. It can generate a sine wave, square wave, sawtooth wave, triangle wave, and other waves from $1 \mathrm{~Hz}$ to $65 \mathrm{kHz}$. The circuit is capable of generating an amplitude of 0.5-14 Vpp and the output impedance is 20-200 ohms. The waveform and frequency of the signal could be adjusted through the buttons and the LCD screen. The amplitude can be adjusted by the amplitude knob. In the system, a sine signal with a frequency of $1 \mathrm{kHz}$ and an amplitude of $14 \mathrm{Vpp}$ is used as the input signal. $1 \mathrm{kHz}$ is chosen according to the existed research ${ }^{[20]}$, which can improve the measurements marginally. $14 \mathrm{Vpp}$ is the maximum of the DDS circuit's output signal, which can improve the SNR.

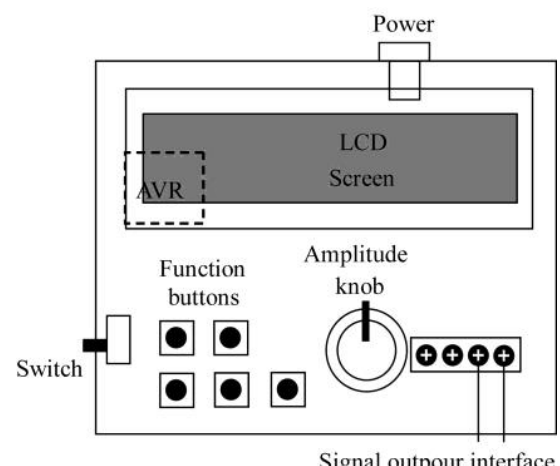

Figure 3 Diagram of DDS signal generation circuit

Compared with the electrical signal generated by the traditional AC analog circuit, the DDS signal generation circuit could generate a variety of waveforms with larger amplitudes and more standard waveforms. The electrical signals generated by the traditional AC signal generation circuit and DDS signal generation circuit are shown in Figure $4 \mathrm{a}$ and Figure $4 \mathrm{~b}$, respectively. It could be seen that the signal in Figure 4a contains a lot of noises and the SNR is low. While the signal in Figure $4 \mathrm{~b}$ has a larger amplitude and SNR than the traditional signal shown in Figure 4a.

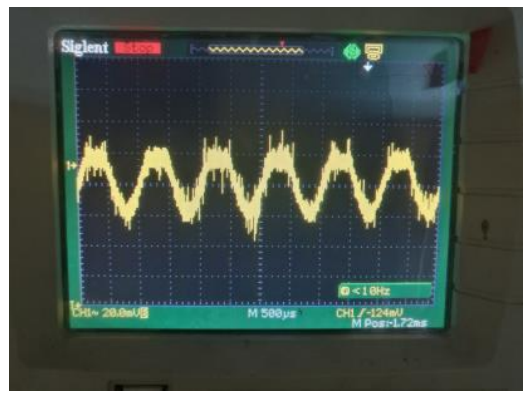

a. Signal generated by traditional signal generation circuit

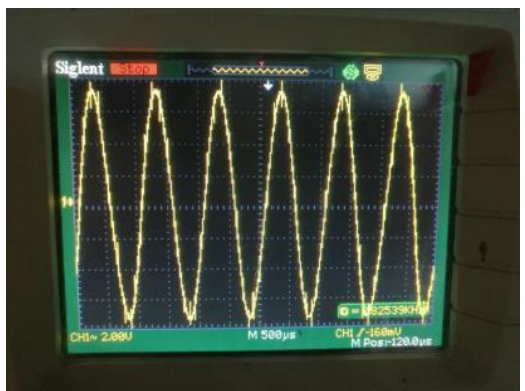

b. Signal generated by DDS signal generation circuit

Figure 4 Comparison of the signal generated by traditional signal generation circuit and DDS signal generation circuit

In the traditional soil EC measurement system, the current of the input signal is assumed to be a constant value, which doesn't change while load varies. In the actual measurement procedure, the current intensity of the input signal will change when the load varies, which will greatly reduce the accuracy of the soil EC measurement system. In order to improve the measurement accuracy of soil EC, the current intensity of the input signal is recorded synchronously with soil output signals, which could eliminate the influence of the input signal's instability on the measurement results.

\subsection{Digital oscilloscope}

The portable digital oscilloscope (DreamSourceLab open-source hardware lab) was used to collect, display and record two channel soil output electrical signals. The soil output signals 
were saved by the oscilloscope along with the environmental noises. By analyzing the data, SNR could be evaluated. The closer the output signals are to the input signal (sine waveform), the less the output signals are affected by noises.

As shown in Figure 5, the digital oscilloscope is small (103 $\mathrm{mm} \times 74 \mathrm{~mm} \times 13 \mathrm{~mm}$ ), easy to carry and integrate. The input voltage is $-100-100 \mathrm{~V}$ and the aluminum alloy case can shield the influences of the environmental noises. The oscilloscope processes signal based on FPGA. The bandwidth is $50 \mathrm{MHz}$ and the maximum sampling frequency is $200 \mathrm{MHz}$. With the software of the oscilloscope, two channels of soil output signals could be collected. The oscilloscope could display real-time waveforms, storage waveforms and display spectrograms.

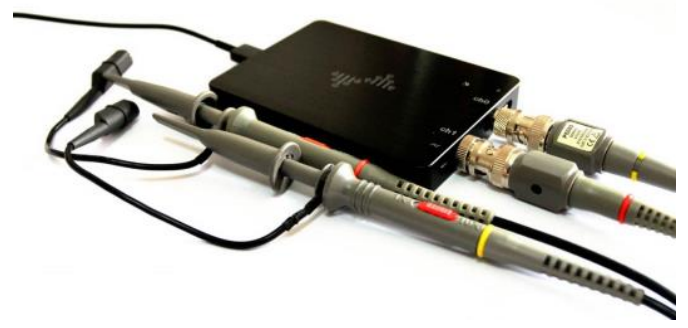

Figure 5 Digital oscilloscope

\subsection{Digital filter}

The soil output signals and the environmental noises are recorded by the digital oscilloscope and saved in PC as CSV files. In order to reduce the influence of environmental noises and improve the measurement accuracy of soil EC, a digital bandpass filter based on Chebyshev type I digital filter was applied. The bandpasses of the filter range are from $990 \mathrm{~Hz}$ to $1100 \mathrm{~Hz}$. And the frequency of the input signal was $1 \mathrm{kHz}$. Except for 990$1100 \mathrm{~Hz}$, the noises of all frequencies are filtered out, which could reduce the influence of the environmental noises in the farmland.

The output signals were converted into frequency domain when filtering. As shown in Figure 6a, the main noises were harmonics. The noises of other frequencies were relatively small. The filtered signal spectrum is shown in Figure $6 \mathrm{~b}$. The voltage at $1 \mathrm{kHz}$ before filtering and after filtering is $886.92 \mathrm{mV}$ and $864.42 \mathrm{mV}$ respectively. The signal intensity at $1 \mathrm{kHz}$ is less affected by the filter and the noises are eliminated obviously.

\subsection{Soil samples}

In order to verify the stability and repeatability of the system, a series of tests were carried out. 108 measurements were conducted by the developed soil EC system at the Shangzhuang Experimental Station of China Agricultural University, Beijing, and 108 soil samples were collected from each experimental position. Those soil samples were sealed in light-shielded sealed bags to minimize the impacts of the environment. And then the soil EC value of the 108 soil samples was also measured with the laboratory solution measurement method respectively. Corn was cropped in the experimental field for at least seven years. The field was under proper management after corn was planted and left idle after corn was harvest. For the 108 soil samples, the moisture varied from $6.3 \%$ to $15.6 \%$. For soil texture, the clay fraction is $34.5 \%$, the silt fraction is $56.4 \%$ and the sand content is $9.1 \%$. The nitrogen content is $0.1328 \mathrm{mg} / \mathrm{kg}$.

In order to verify the application prospects of the system, 10 soil samples with different nitrogen contents were also made and the measurements of soil EC were measured using the developed system and the laboratory method. For these 10 prepared soil samples, the soil moisture is $10.6 \%$, the clay fraction is $34.3 \%$, the silt fraction is $56.8 \%$, and the sand content is $8.9 \%$.

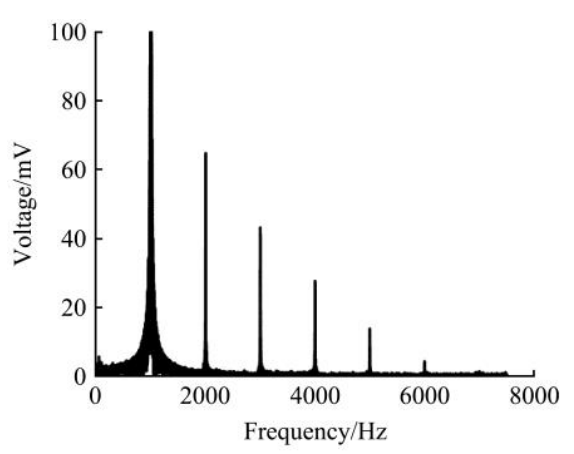

a. Before filtering

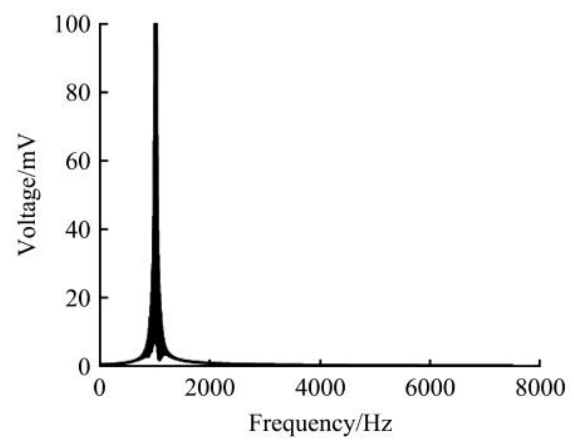

b. After filtering

Figure 6 Comparison of frequency domain diagram before and after filtering

\section{Results and discussion}

\subsection{Waveform of output signals before and after filtering}

The waveform comparison of the soil output signals before and after filtering is shown in Figures $7 \mathrm{a}$ and $7 \mathrm{~b}$ respectively. Due to noises interference, the waveform fluctuated significantly at the peaks and troughs before filtering, and it became approximately standard sine wave after filtering. As seen in Figure 7, the digital filter achieved a very good filtering effect.
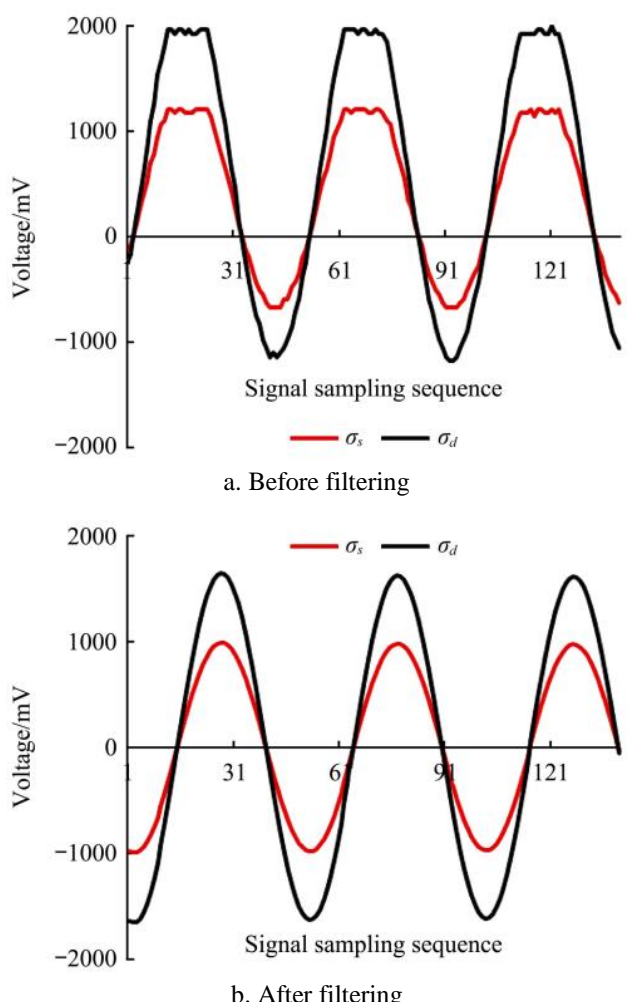

Figure 7 Comparison of waveform diagram before and after filtering 
A part of the filtered signal waveform is shown in Figure 8. The first several waveforms distorted. And the next 5 waveforms have a slightly larger amplitude. The following waveforms become smooth. Therefore, the first 20 waveforms are deleted to eliminate the influences of the waveform distortion after filtering. Then the average value of the peaks and troughs are calculated separately. The formula for calculating the voltage effective value of soil output signals is shown as the following:

$$
V_{\text {Valid }}=\frac{\sum_{i=1}^{n}\left(V_{\text {Peak }}-V_{\text {Trough }}\right)}{2 \sqrt{2} n}
$$

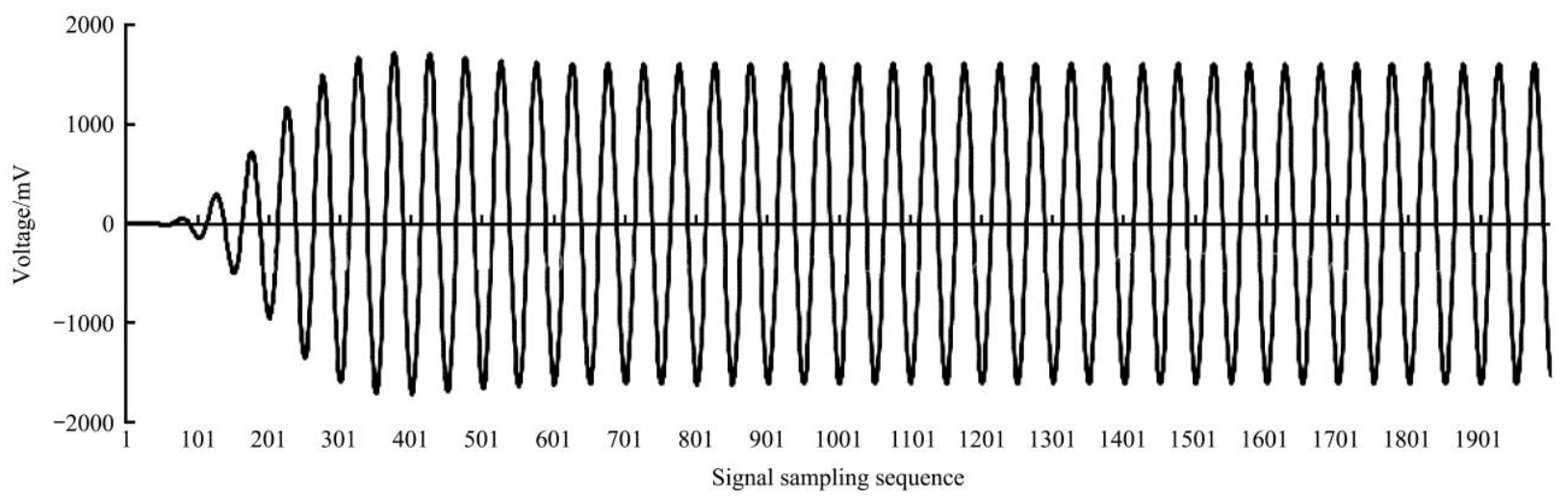

Figure 8 Part of the waveform diagram after filtering of the soil output signals

After calculating the voltage effective value of soil output signals, the corresponding soil EC value could be calculated according to Equations (1) and (2). In the developed system, the internal and external soil output signals were measured simultaneously and the $\sigma_{s}$ and $\sigma_{d}$ were calculated respectively. The average value of $\sigma_{s}$ and $\sigma_{d}$ was taken as the final measurement result of soil EC, which could reduce the impact of soil EC unevenness.

\subsection{System performance experiment}

The corresponding current values of the input signal, two-channel soil output signals were recorded and soil EC values were calculated and analyzed of the 108 field measurements. The average, maximum, minimum and Coefficient of Variation (CV) of the input signal current values are shown in Table 1. It could be seen from the table that the current value varies a lot while the load changes. It's very necessary to measure the current value with the soil output signals synchronously. Otherwise, the instability of the input signal would affect the measurement accuracy seriously.

Table 1 Variability of the current generated by DDS signal generating circuit

\begin{tabular}{ccccc}
\hline & Mean & Min & Max & CV \\
\hline $\mathrm{I} / \mathrm{mA}$ & 0.42 & 0.11 & 0.89 & 38.10
\end{tabular}

Repeatability is very important to the application of the system. In order to evaluate the repeatability of the system, 450 measurements were repeated for the same position in the field experiment. The average, maximum, minimum and $\mathrm{CV}$ of the measurement result are shown in Table 2. It could be seen that the system worked very stably and the fluctuation of the measurement results was small.

Table 2 Stability of soil EC measurements

\begin{tabular}{ccccc}
\hline & Mean & Min & Max & CV \\
\hline $\mathrm{EC} / \mu \mathrm{S} \cdot \mathrm{cm}^{-1}$ & 21.86 & 21.62 & 22.24 & 0.59 \\
\hline
\end{tabular}

When soil texture is even, $\sigma_{s}$ and $\sigma_{d}$ should be highly related. In the 450 sets of $\sigma_{s}$ and $\sigma_{d}$ results of the repeatability experiment, the correlation coefficient of them is 0.91. For the 108 field measurements, the correlation coefficient of $\sigma_{s}$ and $\sigma_{d}$ is 0.93 . It shows that $\sigma_{s}$ and $\sigma_{d}$ are highly related.
The 108 soil samples were also measured for soil EC values in the laboratory using the solution method. The soil samples were dried in a $60^{\circ} \mathrm{C}$ oven for $24 \mathrm{~h}$ firstly. The soil solution was made with a soil to water ratio of 1:5. The solution was fully shaken and then left aside still for 24 hours. The soil EC was measured using a DDB-303A portable conductivity meter (Shanghai Yidian Scientific Instrument Co. Ltd.). The average, maximum, minimum and $\mathrm{CV}$ of the results measured by the developed system in the field experiment and DDB-3-3A meter in the laboratory are shown in Table 3.

Table 3 Comparison of EC measured by developed system and solution method

\begin{tabular}{ccccc}
\hline $\mathrm{EC} / \mu \mathrm{S} \cdot \mathrm{cm}^{-1}$ & Mean & Min & Max & $\mathrm{CV}$ \\
\hline Developed system & 175.95 & 83.99 & 268.21 & 22.10 \\
Solution method & 123 & 77.5 & 299 & 25.73 \\
\hline
\end{tabular}

For the measurement results of these 108 soil EC measurements, the correlation coefficient between the developed system and the solution method is 0.7 . The correlation coefficient is not very high. It's mainly because the laboratory solution measurement method eliminates the influence of soil moisture, soil texture and other factors. The developed system measurement needs to further eliminate the effect of those factors.

\subsection{Prediction of soil nitrogen content using the developed system}

Nitrogen is an essential element for crop growth. Rapid measurement of soil nitrogen content is very important for Precision Agriculture ${ }^{[21]}$. In this study, 10 soil samples with $\mathrm{KNO}_{3}$ content of $0.33 \%, 0.67 \%, 1.00 \%, 1.33 \%, 1.67 \%, 2.00 \%$, $2.33 \%, 2.67 \%, 3.00 \%$ and $3.33 \%$ were prepared. The soil sample was pulverized, sieved and thoroughly stirred and divided into 10 portions. After calculating the weight of $\mathrm{KNO}_{3}$ needed for each portion of the soil sample, each $\mathrm{KNO}_{3}$ sample was dissolved in an equal amount of distilled water, and mixed with each soil sample and stirred thoroughly. Finally, the soil samples mixed with $\mathrm{KNO}_{3}$ samples were air dried to a suitable level and measured with the developed system after fully stirring again.

The soil EC of these 10 soil samples were measured with the developed system. The relationship between soil EC and soil $\mathrm{KNO}_{3}$ content was analyzed and shown in Figure 9. The accuracy 
of the prediction is 0.93 , which has achieved a good prediction accuracy. Under the conditions of consistent soil parameters such as soil moisture, soil particle size and temperature, there was a strong correlation between soil EC and soil nitrogen content. Soil nitrogen content could be predicted with soil EC when other soil parameters are consistent.

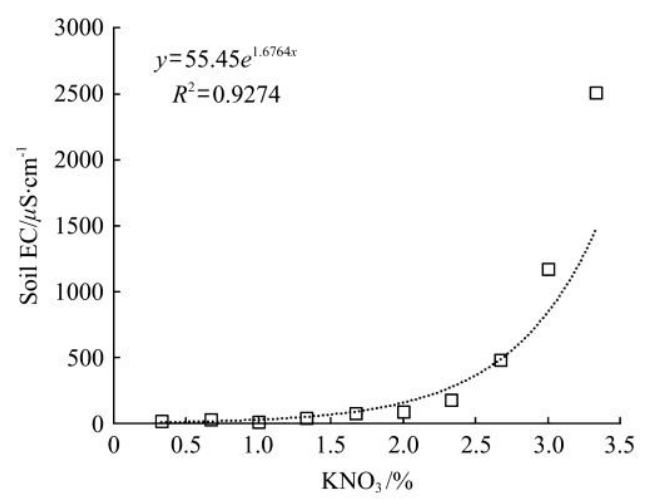

Figure 9 Relationship of soil EC and soil Nitrogen content

\subsection{Discussion}

Soil EC is related to a variety of soil parameters. Soil EC measurement could be used to reflect the soil conditions, which is of great significance to Precision Agriculture ${ }^{[22,23]}$. For the traditional soil EC measurement system based on the "current-voltage four-electrode method", the input signal was unstable. The electrical signals were easily affected by the environment. It was hard to evaluate the SNR of the soil output signals and the effect of the digital filter. Only a small amount of information could be recorded.

In response to the above disadvantages, a soil EC measurement system based on the DDS and digital oscilloscope was developed. In the developed system, DDS signal generation circuit was used to provide a more standard input signal. The current value of the input signal was recorded with soil output signals to eliminate the instability of the input signal, which varied with the change of load. Two-channel soil output signals were recorded along with the environment noises by the digital oscilloscope. And it was able to record the detailed signals in microseconds. After filtering by the digital filter, the noises of all frequencies except for $990-1100 \mathrm{~Hz}$ were filtered out, which greatly suppressed the influences of the noises and improve the SNR of the signals. The filtered waveform is approximately a standard sine waveform. The voltage effective value of the soil output signal is calculated by averaging, which could reduce the influence of noises further. $\sigma_{s}$ and $\sigma_{d}$ could be calculated with Equations (1) and (2). By calculating the average of $\sigma_{s}$ and $\sigma_{d}$, the influence of soil texture inhomogeneity on soil EC was further reduced.

The stability of the input signal and the overall measurement stability of the system were analyzed through experiments. And the measurement results of the developed system were compared with the measurement results of the laboratory solution method. Besides, soil samples with different nitrogen contents were prepared and the soil EC of them was measured by the developed system. It achieves a good accuracy when predicting soil nitrogen content with soil EC measurements. In the case of consistent soil moisture, soil texture, soil temperature and other parameters, soil EC could be used to predict soil nitrogen content accurately.

The results above mentioned were basically achieved under the conditions of the laboratory. Although it has proved the performance of the developed system, it is necessary to conduct field tests in the next study to improve practicability. Furthermore, it is necessary to study the new algorithms of processing the oscilloscope's waveforms in the future. With proper data processes algorithms, the impacts of complex farmland environment could be reduced and the accuracy and application range of the system could be improved.

\section{Conclusions}

1) A DDS signal generation circuit was used to generate an input signal, which significantly improves the stability of the input signal. It could reduce the influence of environment noises on the input signal and provide a standardized sinusoidal signal. The current value of the input signal and the soil output signals were recorded simultaneously. It could eliminate the influence of instability of the input signal on the measurement accuracy completely.

2) Two-channel soil output signals mixed with the environment noises were recorded by the digital oscilloscope in microseconds, which could record the detailed information of the signals. The waveform of these signals could be seen directly on the PC to evaluate the SNR of the output signals. When the SNR significantly reduced, system adjustment was needed to improve the SNR. The detailed waveform data could be used to analysis the reason for waveform jitter and noise enhancement, which could improve measurement accuracy further.

3) The use of the bandpass digital filter significantly improved the filtering effect. It could filter the noises of all frequencies except for the input signal's frequency, which could improve the SNR. The filtered signal was approximately a standard sine wave. The filtering effect of the digital filter could be intuitively evaluated by comparing the signal waveforms before and after filtering. When the filtering effect reduced, the parameters of the digital filter could be adjusted to improve the filtering effect.

4) A large number of waveforms were recorded by the digital oscilloscope. And the effective values of the soil output signals were calculated by averaging, which could reduce the influence of the environment noises. The corresponding $\sigma_{s}$ and $\sigma_{d}$ were calculated. By calculating the average of the $\sigma_{s}$ and $\sigma_{d}$, the influence of soil texture unevenness could be further reduced to improve the system measurement accuracy.

\section{Acknowledgements}

This study was supported by the Chinese National Key Research and Development Plan (2016YFD0700300 -2016YFD0700304) and the National Natural Science Foundation of China (31801265).

\section{[References]}

[1] De Baerdemaeker J. Precision agriculture technology and robotics for good agricultural practices. IFAC Proceedings, IFAC, 2013; 46: 1-4.

[2] Monzon J P, Calviño P A, Sadras V O, Zubiaurre J B, Andrade F H. Precision agriculture based on crop physiological principles improves whole-farm yield and profit: A case study. Eur J Agron, 2018; 99(6): 62-71.

[3] Finch H J S, Samuel A M, Lane G P F. Precision farming. In: Lockhart \& Wiseman's Crop Husbandry Including Grassland. 9th ed. 2014; pp.235-244.

[4] Lan Y B, Chen S D, Fritz B K. Current status and future trends of precision agricultural aviation technologies. Int J Agric Biol Eng. 2017; 10(3): 1-17

[5] Varella C A A V, Gleriani J M, Santos R M D. Precision agriculture and remote sensing. In: Fernando S, Aluízio B, Celso C, editors. Sugarcane: 
Agricultural Production, Bioenergy and Ethanol. Elsevier Inc; 2015; pp.185-203.

[6] Reuter H I, Kersebaum K C. Applications in precision agriculture. In: Developments in Soil Science, 2018; pp.623-36.

[7] Sabarina K, Priya N. Lowering data dimensionality in big data for the benefit of precision agriculture. Procedia Comput Sci [Internet]. 2015; 48(C): 548-554. http://dx.doi.org/10.1016/j.procs.2015.04.134

[8] Yin Y, Chen L, Meng Z, Li B, Luo C, Fu W, et al. Design and evaluation of a maize monitoring system for precision planting. Int J Agric Biol Eng, 2018; 11(4): 166-170.

[9] Li M Z, Wang M H, Wang Q. Development and performance test of a portable soil EC detector. Appl Eng Agric, 2006; 22(2): 301-307.

[10] Yuan C F, Feng S Y, Wang J, Huo Z L, Ji Q Y. Effects of irrigation water salinity on soil salt content distribution, soil physical properties and water use efficiency of maize for seed production in arid Northwest China. Int J Agric Biol Eng, 2018; 11(3): 137-145.

[11] Calixto W P, Martins Neto L, Wu M, Kliemann H J, de Castro S S, Yamanaka K. Calculation of soil electrical conductivity using a genetic algorithm. Comput Electron Agric, 2010; 71(1): 1-6.

[12] Corwin D L, Lesch S M. Apparent soil electrical conductivity measurements in agriculture. Comput Electron Agric. 2005; 46(1-3 SPEC. ISS.): $11-43$.

[13] Sudduth K A, Kitchen N R, Drummond S T. Soil conductivity sensing on claypan soils: comparison of electromagnetic induction and direct methods. Proceedings of 4th International Conference on Precision Agriculture, 1998; pp.979-990.

[14] Eric D. Lund, Colin D. Christy, Paul E. Drummond. using yield and soil electrical conductivity (EC) maps to derive crop production performance information. Presented at the 5th International Conference on Precision Agriculture, 2005.

[15] Pei X, Zheng L, Li M, Sun H. Development of a vehicular soil electrical conductivity monitoring system based on ARM. Am Soc Agric Biol Eng Annu Int Meet 2014, ASABE, 2014; 3: 1747-56.

[16] Zhang J, Li M, Kong D. Measurement of substrate electrical conductivity in greenhouse based on electrical current-voltage four-electrode method. J Jilin Univ Eng Technol Ed, 2007; 37(2): 484-488.

[17] Chen L, Li M, Zhao Y. Improvement and experiment of the portable soil EC detector. J Agric Mech Res. 2009;7: 175-177.

[18] Li M, Kong D, Zhang J, Sui W, Zou Q Z. Development of portable soil EC meter with Bluetooth and PDA. J Jiangsu Univ Nat Sci Ed. 2008; 29(2): 93-96.

[19] Direct digital synthesis - Wikipedia. https://en.wikipedia.org/wiki/ Direct_digital_synthesizer.

[20] Robinson D A, Kelleners T J, Cooper J D, Gardner C M K, Wilson P, Lebron I, et al. Evaluation of a capacitance probe frequency response model accounting for bulk electrical conductivity. Vadose Zo J, 2005; 4(4): 992.

[21] An X, Li M, Zheng L, Liu Y, Sun H. Effect of soil moisture on prediction of soil total nitrogen using NIR spectroscopy. Spectrosc Spectr Anal, 2013; 33(3): 677-681.

[22] Sudduth K A, Drummond S T, Kitchen N R. Accuracy issues in electromagnetic induction sensing of soil electrical conductivity for precision agriculture. Comput Electron Agric, 2001; 31(3): 239-264.

[23] Cho Y, Sudduth K A, Chung S O. Soil physical property estimation from soil strength and apparent electrical conductivity sensor data. Biosyst Eng, 2016; 152: 68-78. 